\title{
Reseñas - Comptes-rendus - Reviews
}

Quaghebeur, Marc, (2015) Histoire, Forme et Sens en littérature. La Belgique francophone. Tome 1: L'engendrement (1815-1914). P.I.E. Peter Lang, collection Documents pour l'Histoire des Francophonies - Théorie, vol. 40, 430 pp., ISBN : 978-2-87574-276-6.

Mots clés : Belgique francophone, $\mathrm{XIX}^{\mathrm{e}}$ siècle, Histoire, Sens, Forme, Mythes, Identité, Langue

Charles De Coster, A. Nirep, Francis Nautet, Edmond Picard, Emile Verhaeren, Maurice Maeterlinck, Albert Mockel, Jules Destrée, Rosny aîné, Georges Eekhoud, Maria Van Rysselberghe... Ces quelques noms emblématiques présents dans la «Table des matières » indiquent d'emblée que ce premier tome couvre une période ample du champ littéraire francophone belge et essentielle dans la composition de celui-ci. Un siècle exactement, depuis la déroute napoléonienne à Waterloo en juin 1815 - et le passage consécutif des territoires belges sous la coupe hollandaise à la suite du Congrès de Vienne, et ce pour une quinzaine d'années au cours desquelles se manifestent toute une série d'éléments discursifs et mythiques qui nourriront cette littérature - jusqu'au début de la Grande Guerre, voire un peu au-delà.

Une fois encore, et pour notre plus grand plaisir de lecteur, Marc Quaghebeur a décidé de rebrasser une matière qu'il maîtrise avec excellence et de nous fournir, dans ce volume de plus de 400 pages et dont l'illustration de couverture est un fragment du $\mathrm{Feu}$ d'artifice (1887) de James Ensor, une version actualisée et complétée de nombre de ses études plus anciennes.

Comme l'auteur l'indique dans son «Avant-propos » et dans sa «Préface », le titre générique de l'ouvrage - Histoire, Forme et Sens - permet d'en dégager les lignes de faîte: l'enracinement et l'articulation des faits littéraires dans et à l'Histoire, quel que soit le niveau, un lien sans cesse mis en évidence par les chefsd'œuvre du corpus, lesquels demeurent néanmoins incompréhensibles en dehors de l'espace dans lequel ils virent le jour et au sein duquel ils font Signe et Sens. Car, précise-t-il, « le corpus littéraire belge de langue française du XIX ${ }^{\mathrm{e}}$ siècle est le fruit d'une Histoire singulière et le terreau de certaines Formes esthétiques qui firent date et dont l'articulation avec Elle fait Sens. Ces trois termes, ceux du titre de l'ouvrage, sont donc écrits en majuscules à chacune de leurs occurrences » (p. 20).

L'ouvrage comprend sept chapitres aux titres destinés à piquer la curiosité du lecteur et qui indiquent l'originalité de la démarche et de la méthode quaghebeuriennes.

(1) «S'inscrire au cœur du légendaire », le premier chapitre de ce premier tome, outre qu'il analyse quelques-unes des figures et œuvres emblématiques du XIX ${ }^{\mathrm{e}}$ siècle, dévoile et explicite l'ensemble du processus littéraire francophone qui s'est déployé en Belgique dès 1815. C'est dire que, tout en pointant quelques-unes des 
particularités, persistantes ou mouvantes du corpus dont il est ici question, à l'aune des amples séquences temporelles qui rythment ces deux siècles de vie littéraire (1815-2015), il ancre dans l'Histoire même du royaume de Belgique certaines des spécificités offertes par cette première littérature non française mais écrite en langue française. Cette originalité par rapport au champ littéraire français et à sa conception jacobine de la langue, de l'identité et de la littérature explique donc, parmi d'autres faits, pourquoi - comme l'indique le sous-titre de ce chapitre : «Aux confins du réel ; au bord du fantastique » - quantité d'œuvres s'y sont développées, en marge du strict réel, dans les territoires propres au légendaire et au fantastique.

La première œuvre importante du corpus, qui, dès son titre atypique : $L a$ Légende et les Aventures hérö̈ques, joyeuses et glorieuses d'Ulenspiegel et de Lamme Goedzak au pays de Flandres et ailleurs (1867) et sa « Préface du Hibou » à la deuxième édition (1869), annonce un non-conformisme aux normes parisiennes, n'en est-elle pas la meilleure illustration ? Assurément De Coster, défini par Quaghebeur comme «le premier romancier francophone de l'histoire de la langue française » (p. 29), ouvre une veine dans laquelle s'engouffreront avec plaisir et talent maints écrivains de son pays, notamment ceux de la grande époque de la fin du $\mathrm{XIX}^{\mathrm{e}}$ siècle, une époque prospère marquée par l'entreprise coloniale menée à bien par le roi Léopold II et à laquelle mettront symboliquement et dramatiquement fin l'invasion et l'occupation allemandes en 1914. Cet événement majeur sera en effet à l'origine de la mise à mort de plusieurs mythes, tel celui du mythe germanolatin censé définir le Belge et sa littérature, et d'une réorientation de la littérature en langue française produite par les écrivains de Belgique : cap sur la France, tel que le préconiseront les signataires du Manifeste du lundi (1937). Mais les différences se s'effacent pas d'un coup de plume : après avoir mis le doigt sur les spécificités importantes des surréalismes français et belges (à Bruxelles et dans le Hainaut), Quaghebeur pose avec pertinence la question suivante: "Au beau milieu de la Seconde Guerre mondiale et particulièrement au tournant de la guerre, qu'écrit par exemple Michel de Ghelderode ?» (p.43).

Examinant les conséquences de ce nouveau conflit dans une Belgique plongée dans sa "Question royale», Quaghebeur constate que ces années difficiles n'engendreront alors que peu de textes fictionnels et déboucheront sur le triomphe d'une « esthétique néoclassique » et l'incarnation de valeurs plutôt intemporelles ; autrement dit, cette période, qui s'étend du lendemain de la Grande Guerre à la fin des années 1960, est globalement celle de la dénégation de l'historicité propre. C'est qu'il faudra attendre la génération suivante, celle de la « Belgitude » (1976) et du Manifeste pour la culture wallonne (1983), qui se développe principalement à partir de la décennie 1970, pour voir enfin certains de ces sujets délicats, voire tabous jusqu'alors, être traités dans toute leur complexité. Cependant, si certains romanciers ont recours aux canons réalistes, la plupart préfèrent passer par « une sorte de crépusculisme [qui] leur sert de tonalité englobante », non seulement pour se distinguer de la Littérature française «mais aussi pour y dire et insérer des formes d'historicité qui ne se moulent pas dans les canons français dominants » (p. 
49). Il est vrai que la fiction belge de langue française offre une place de choix aux anti-héros !

(2) «Se figurer à l'heure des littératures nationales ", le deuxième chapitre s'intéresse aux mythes qui, dès avant la proclamation de l'indépendance belge, vont irriguer cette littérature et permettre, du moins pendant près d'un siècle, l'apparition de chefs-d'œuvre clairement identifiables et distincts de ce qui est produit en France à la même époque.

Il va de soi que les ingrédients historiques occupent une place centrale dans la confection de ce réseau mythique, en particulier ce que Quaghebeur identifie comme le Siècle d'or de ces territoires, à savoir la période qui va grosso modo de 1450 à 1560 c'est-à-dire l'époque des Pays-Bas bourguignons (tout spécialement avec les figures tragiques de Charles le Téméraire et de sa fille Marie de Bourgogne) et espagnols (avec celles de Charles Quint, si chère à l'auteur, et de son fils Philippe II). De fait, l'évocation de ces $\mathrm{XV}^{\mathrm{e}}$ et $\mathrm{XVI}^{\mathrm{e}}$ siècles occupe un espace de choix dans le corpus littéraire francophone belge dès avant même 1830, et tout au long du XX siècle. Assurément, dans ce puissant «mythe fondateur» du $\mathrm{XVI}^{\mathrm{e}}$ siècle qui non seulement véhicule les idéaux de liberté et de prospérité mais fonctionne sur une logique manichéenne, l'Espagne constitue « un Autre univoquement négatif [...], l'incarnation du désastre, de l'oppression et de la tyrannie infligés à un peuple qui ne demandait qu'à vivre et à jouir » (p. 70).

Dans la deuxième partie de ce chapitre, Quaghebeur analyse les «avatars et la permanence » du mythe jusque dans les dernières décennies pour constater notamment que, dans les productions plus récentes, « si l'image de l'Espagne noire ne disparaît pas forcément, elle ne prend plus les connotations exacerbées des textes héroïsants et manichéistes de la constitution des identités nationales » (p. 143-144).

(3) «Se vivre à travers l'Europe », le troisième chapitre nous révèle le rôle joué par «Bruxelles, ville d'accueil, creuset d'une littérature», une périphérie accueillante pour nombre de ceux qui, pour des raisons essentiellement politiques et principalement au cours de la seconde moitié du $\mathrm{XIX}^{\mathrm{e}}$ siècle, durent fuir Paris et participèrent d'une façon ou d'une autre à encourager l'éclosion et le développement du champ littéraire belge. Il analyse aussi le jeu dialectique singulier dans lequel la capitale belge, de par sa proximité géographique et l'usage croissant du français (certes particulier !), se positionne par rapport à la VilleLumière, laquelle, au tournant des deux siècles, reçoit à bras ouverts de nombreux écrivains - notamment quelques «exotiques » francophones de Flandre - dont elle assure le rayonnement international. A l'époque, Bruxelles, que les grands travaux léopoldiens transforment en capitale moderne, devient un authentique «carrefour de pensée européenne » (p. 162), d'autant plus que son activité intellectuelle couvre l'ensemble des connaissances relatives à un monde en pleine expansion.

(4) «S'inventer entre historisation et carnavalisation », le quatrième chapitre part du pas fort audacieux mais décisif fait en 1867 par De Coster qui, en publiant un roman baroque, hors normes et hors genres, permit, à la surprise et au désagrément de ceux qui avaient intériorisé comme valeur indiscutable le fonctionnement du système culturel et identitaire de la France, d'ouvrir en Belgique 
la voie d'une production littéraire spécifique en langue française. Si De Coster n'en tira aucun gain de son vivant, sa Légende fut cependant célébrée par la génération de 1880 car elle «accomplissait dans la Forme et dans la Langue le mythe et l'espérance de ces écrivains » (p. 199).

Vingt ans plus tard, en 1887, A. Nirep publie Les mystères du Congo, un roman qui relate, lui aussi sur le mode carnavalesque, l'histoire de la colonisation belge en Afrique et met en scène un personnage qui, né un jour de carnaval et se nommant de Spiegel, apparait donc, mutatis mutandis, comme « un curieux pendant colonial » (p. 200) du héros de De Coster. Comme le note Quaghebeur, bien qu'elle ait été une pièce clé dans l'histoire de la Belgique, l'Afrique centrale n'occupe qu'une place secondaire dans le corpus «noble » des Lettres belges de langue française. C'est que la plupart des auteurs des textes mettant en scène ce continent avec lequel ils ont généralement un rapport direct ne font pas partie du milieu littéraire proprement dit et se doivent de recourir à des canaux d'édition spécifiques. Tel fut le cas de Nirep (anagramme de Périn) dont les objectifs - «la diffusion et la légitimation de l'aventure africaine de Léopold II (p. 229) - étaient limpides et dont le « grand roman d'aventures », qui peut être considéré comme « la fiction de la conquête » (p. 228), est ici analysé dans toute sa richesse.

(5) «S'écrire dans une langue à soi », le cinquième chapitre se centre sur cette période où différents mythes (le $\mathrm{XVI}^{\mathrm{e}}$ siècle, la Flandre littéraire et picturale, la synthèse germano-latine) permettent à toute une génération d'écrivains symbolistes ou naturalistes, la plupart issus de Flandre, de produire en français des œuvres, voire des chefs-d'œuvre, d'une profonde originalité à un moment où leur pays brille de tous ses feux.

C'est aussi à cette époque qu'apparaissent les premiers essais qui tentent de cerner les spécificités de cette nouvelle littérature que Francis Nautet, dans une première tentative globale d'historiographie de la production en langue romane de la Belgique - une synthèse «elle-même foncièrement mythique » (p. 258) - nomme "lettres belges d'expression française », et ce pour la différencier clairement de la littérature flamande qui éclot alors. A côté des avocats et essayistes bruxellois et wallon tels que Edmond Picard ou Albert Mockel qui, dans leurs revues ou ailleurs, animèrent les débats, des figures aussi emblématiques que celles de Maeterlinck et de Verhaeren déployèrent une activité métadiscursive nullement négligeable. Dans les pages qui suivent, Quaghebeur visite le «premier laboratoire » que constitue $L e$ Cahier bleu de l'auteur de Pelléas et Mélisande avant de relire, dans des pages qu'il intitule «Le pictural comme métaphore du combat pour la littérature », la monographie que le critique d'art et poète des Villes tentaculaires consacra à James Ensor.

(6) « Se lire au tournant du siècle », le sixième chapitre s'attache à commenter deux œuvres qui, au dire du critique, contiennent des indices du rapport particulier à l'Histoire et à la conscience de soi qui caractérise nombre des auteurs francophones belges : L'Intelligence des fleurs (1907) de Maeterlinck et La force mystérieuse (1912-1913 en feuilleton ; 1914 en volume) de J.-H. Rosny aîné (plus connu pour La Guerre du feu). 
Tout en révélant qu'un même fil imaginaire sous-tend de façon cohérente toute l'œuvre - «de la scène à la flore » - et l'univers idéaliste du Prix Nobel de littérature 1911, le premier texte recèle quantité de motifs qui «lui donnent une portée qui dépasse celle de la pure description botanique » (p. 342) et témoigne d'une civilisation en pleine déliquescence.

Quant au second texte, qui appartient à un genre généralement situé dans la littérature populaire ou dans la «paralittérature » et dont le titre - renvoyant à la science-fiction - constitue à plus d'un égard un «symptôme d'époque » (p. 353), il annonce que la prompte résurgence des pires instincts et des plus effroyables pulsions de l'homme vouera cette civilisation gangrénée à une extinction violente. Si c'est à Paris, véritable poudrière sociale, que la catastrophe se déclenche, c'est bientôt la planète entière qui en subit le brutal impact... Le double finale, «celui de l'amour et de la science» (p. 370), n'adviendra que lorsqu'une bonne part de la population mondiale aura été décimée, que les villes auront retrouvé des proportions plus humaines... Deux ans à peine après la parution du premier épisode, le continent européen sera mis à feu et à sang.

(7) Comme son titre l'indique - «Se dire après le désastre »-, le septième et dernier chapitre de ce premier tome constitue une sorte de transition : y sont en effet analysés deux ouvrages publiés le premier en 1928, le second en 1934 : Magrice en Flandre ou le buisson des mendiants et Il y a 40 ans, dont les auteurs respectifs, Georges Eekhoud et Maria Van Rysselberghe, appartiennent en plein à la génération léopoldienne dont ils furent des acteurs culturels engagés.

Dans son «roman picaro-chevaleresque » (tel est son sous-titre), celui que Nautet considérait comme l'emblème de la littérature nouvelle a convoqué, à la veille de sa mort en 1927 et à travers le regard d'un chevalier portugais, donc d'un homme venu d'un autre petit pays du Sud, «tous les éléments du mythe [nordique et de l'âme belge] sur lequel vécut la première grande génération littéraire francophone non française » (p. 373). C'est donc à travers le contraste des caractères et tempéraments méridional et septentrional que l'écrivain anversois s'emploie à exalter le «pays de cocagne et univers pictural » que fut censé être la Belgique d'antan et de naguère: un monde culturellement singulier (au sein de l'Europe et par rapport à la France), qui venait, quelques années auparavant, de ratifier plusieurs des qualités fantasmées - héroïsme, loyauté, noblesse, courage, générosité, pacifisme, amour de la liberté... - qui cimentèrent son mythe fondateur.

Dans son récit publié sous un pseudonyme lourd de sens - celui de M. SaintClair -, la femme du peintre Théo Van Rysselberghe, outre qu'elle remémore la passion intense et pudique qu'elle ressentit pour celui qui, dès sa mort accidentelle à Rouen en 1916, incarna la figure du poète national (Verhaeren), révèle aussi « ce que fut le combat pour - et l'amour de - l'Art, la Littérature et la Lumière » (p. 374) chez ce cercle d'écrivains et d'artistes qui s'efforcèrent de placer la Belgique au centre de l'échiquier culturel européen. C'est dire que le côté autobiographique est constamment présent dans ce texte à clés et écrit dans une langue un brin classique, celle que beaucoup d'écrivains belges de l'après-Première Guerre admirent déjà et revendiqueront très bientôt de manière officielle. Pas de doute pour Quaghebeur, 
cette femme unique et passionnée fut une des plus belles plumes produites par la Belgique du XIX ${ }^{\mathrm{e}}$ siècle.

André BÉNIT

Universidad Autónoma de Madrid andre.benit@uam.es 